\title{
An interesting class of Hankel determinants
}

\author{
Johann Cigler* \& Mike Tyson ${ }^{\dagger}$
}

\begin{abstract}
For small $r$ the Hankel determinants $d_{r}(n)$ of the sequence $\left(\left(\begin{array}{c}2 n+r \\ n\end{array}\right)\right)_{n \geq 0}$ are easy to guess and show an interesting modular pattern. For arbitrary $r$ and $n$ no closed formulae are known, but for each positive integer $r$ the special values $d_{r}(r n)$, $d_{r}(r n+1)$, and $d_{r}\left(r n+\left\lfloor\frac{r+1}{2}\right\rfloor\right)$ have nice values which will be proved in this paper.
\end{abstract}

\section{Introduction}

Let $\left(a_{n}\right)_{n \geq 0}$ be a sequence of real numbers with $a_{0}=1$. For each $n$ consider the Hankel determinant

$$
H_{n}=\operatorname{det}\left(a_{i+j}\right)_{i, j=0}^{n-1} .
$$

We are interested in the sequence $\left(H_{n}\right)_{n \geq 0}$ for the sequences $a_{n, r}=\left(\begin{array}{c}2 n+r \\ n\end{array}\right)$ for some $r \in \mathbb{N}$. For $n=0$ we let $H_{0}=1$.

Let

$$
d_{r}(n)=\operatorname{det}\left(\left(\begin{array}{c}
2 i+2 j+r \\
i+j
\end{array}\right)\right)_{i, j=0}^{n-1}
$$

For $r=0$ and $r=1$ these determinants are well known and satisfy $d_{0}(n)=2^{n-1}$ and $d_{1}(n)=1$ for $n>0$. Eğecioğlu, Redmond, and Ryavec [3] computed $d_{2}(n)$ and $d_{3}(n)$ and stated some conjectures for $r>3$.

Many of these determinants are easy to guess and show an interesting modular pattern. For example

\footnotetext{
*Email: johann.cigler@univie.ac.at

$\dagger$ Email:mgtyson66@gmail.com
} 


$$
\begin{aligned}
& \left(d_{0}(n)\right)_{n \geq 0}=\left(1,1,2,2^{2}, 2^{3}, \ldots\right), \\
& \left(d_{1}(n)\right)_{n \geq 0}=(1,1,1,1,1, \ldots), \\
& \left(d_{2}(n)\right)_{n \geq 0}=(1,1,-1,-1,1,1,-1,-1, \ldots), \\
& \left(d_{3}(n)\right)_{n \geq 0}=(1,1,-4,3,3,-8,5,5,-12,7,7,-16, \ldots), \\
& \left(d_{4}(n)\right)_{n \geq 0}=(1,1,-8,8,1,1,-16,16,1,1,-24,24, \ldots), \\
& \left(d_{5}(n)\right)_{n \geq 0}=(1,1,-13,-16,61,9,9,-178,-64,370,25,25,-695,-144,1127, \ldots)
\end{aligned}
$$

These and other computations suggest the following facts:

$$
\begin{aligned}
& d_{2 k+1}((2 k+1) n)=d_{2 k+1}((2 k+1) n+1)=(2 n+1)^{k}, \\
& d_{2 k+1}((2 k+1) n+k+1)=(-1)^{\left(\begin{array}{c}
k+1 \\
2
\end{array}\right)} 4^{k}(n+1)^{k}, \\
& d_{2 k}(2 k n)=d_{2 k}(2 k n+1)=(-1)^{k n}, \\
& d_{2 k}(2 k n+k)=-d_{2 k}(2 k n+k+1)=(-1)^{k n+\left(\begin{array}{c}
k \\
2
\end{array}\right)} 4^{k-1}(n+1)^{k-1} .
\end{aligned}
$$

The purpose of this paper is to prove these conjectures. These methods seem to extend to the Hankel determinants of the sequences $\left(\left(\begin{array}{c}2 n+r \\ n-s\end{array}\right)\right)_{n \geq 0}$, but we do not compute these here.

In Sections 2 and 3 we review some well-known facts from the theory of Hankel determinants. In particular we compute $d_{0}(n)$ and $d_{1}(n)$. In Section 4 we define the matrix $\gamma$ and use it to compute $d_{2}(n)$. In Section 5 we introduce the matrices $\alpha_{n}$ and $\beta_{n}$, which serve as the basis of our method. In Section 6 we write the Hankel matrices in terms of these matrices. In Sections 7 and 8 we use this information to compute $d_{r}(n)$ in the aforementioned seven cases.

We would like to thank Darij Grinberg for his helpful suggestions.

\section{Some background material}

Let us first recall some well-known facts about Hankel determinants (cf. e.g. [1]). If $d_{n}=\operatorname{det}\left(a_{i+j}\right)_{i, j=0}^{n-1} \neq 0$ for each $n$ we can define the polynomials

$$
p_{n}(x)=\frac{1}{d_{n}} \operatorname{det}\left(\begin{array}{ccccc}
a_{0} & a_{1} & \cdots & a_{n-1} & 1 \\
a_{1} & a_{2} & \cdots & a_{n} & x \\
a_{2} & a_{3} & \cdots & a_{n+1} & x^{2} \\
\vdots & & & & \vdots \\
a_{n} & a_{n+1} & \cdots & a_{2 n-1} & x^{n}
\end{array}\right) .
$$


If we define a linear functional $L$ on the polynomials by $L\left(x^{n}\right)=a_{n}$ then $L\left(p_{n} p_{m}\right)=0$ for $n \neq m$ and $L\left(p_{n}^{2}\right) \neq 0$ (orthogonality).

By Favard's Theorem there exist complex numbers $s_{n}$ and $t_{n}$ such that

$$
p_{n}(x)=\left(x-s_{n-1}\right) p_{n-1}(x)-t_{n-2} p_{n-2}(x) .
$$

For arbitrary $s_{n}$ and $t_{n}$ define numbers $a_{n}(j)$ by

$$
\begin{aligned}
& a_{0}(j)=[j=0] \\
& a_{n}(0)=s_{0} a_{n-1}(0)+t_{0} a_{n-1}(1), \\
& a_{n}(j)=a_{n-1}(j-1)+s_{j} a_{n-1}(j)+t_{j} a_{n-1}(j+1) .
\end{aligned}
$$

These numbers satisfy

$$
\sum_{j=0}^{n} a_{n}(j) p_{j}(x)=x^{n}
$$

Let $A_{n}=\left(a_{i}(j)\right)_{i, j=0}^{n-1}$ and $D_{n}$ be the diagonal matrix with entries $d(i, i)=\prod_{j=0}^{i-1} t_{j}$. Then we get

$$
\left(a_{i+j}(0)\right)_{i, j=0}^{n-1}=A_{n} D_{n} A_{n}^{\top}
$$

and

$$
\operatorname{det}\left(a_{i+j}(0)\right)_{i, j=0}^{n-1}=\prod_{i=1}^{n-1} \prod_{j=0}^{i-1} t_{j} .
$$

If we start with the sequence $\left(a_{n}\right)_{n \geq 0}$ and guess $s_{n}$ and $t_{n}$ and if we also can guess $a_{n}(j)$ and show that $a_{n}(0)=a_{n}$ then all our guesses are correct and the Hankel determinant is given by the above formula.

There is a well-known equivalence with continued fractions, so-called J-fractions:

$$
\sum_{n \geq 0} a_{n} x^{n}=\frac{1}{1-s_{0} x-\frac{t_{0} x^{2}}{1-s_{1} x-\frac{t_{1} x^{2}}{1-\ddots}}}
$$

For some sequences this gives a simpler approach to Hankel determinants.

As is well known Hankel determinants are intimately connected with the Catalan numbers $C_{n}=\frac{1}{n+1}\left(\begin{array}{c}2 n \\ n\end{array}\right)$. Consider for example the aerated sequence of Catalan numbers $\left(c_{n}\right)=(1,0,1,0,2,0,5,0,14,0, \ldots)$ defined by $c_{2 n}=C_{n}$ and $c_{2 n+1}=0$. Since the generating function of the Catalan numbers

$$
C(x)=\sum_{n \geq 0} C_{n} x^{n}=\frac{1-\sqrt{1-4 x}}{2 x}
$$

satisfies 


$$
C(x)=1+x C(x)^{2}
$$

we get

$$
C(x)=\frac{1}{1-x C(x)}
$$

and

and therefore

$$
C\left(x^{2}\right)=\frac{1}{1-x^{2} C\left(x^{2}\right)}=\frac{1}{1-\frac{x^{2}}{1-\frac{x^{2}}{1-\ddots}}}
$$

$$
\operatorname{det}\left(c_{i+j}\right)_{i, j=0}^{n-1}=1 .
$$

From $C(x)=1+x C(x)^{2}$ we get $C(x)^{2}=1+2 x C(x)^{2}+x^{2} C(x)^{4}$ or

$$
C(x)^{2}=\frac{1}{1-2 x-x^{2} C(x)^{2}}=\frac{1}{1-2 x-\frac{x^{2}}{1-2 x-\frac{x^{2}}{1-2 x-\ddots}}} .
$$

The generating function of the central binomial coefficients $B_{n}=\left(\begin{array}{c}2 n \\ n\end{array}\right)$ is

$$
B(x)=\sum_{n \geq 0} B_{n} x^{n}=\frac{1}{\sqrt{1-4 x}}=\frac{1}{1-2 x C(x)}=\frac{1}{1-2 x-2 x^{2} C(x)^{2}} .
$$

Therefore by (25) we get the J-fraction

$$
B(x)=\frac{1}{1-2 x-2 x^{2} C(x)^{2}}=\frac{1}{1-2 x-\frac{2 x^{2}}{1-2 x-\frac{x^{2}}{1-2 x-\frac{x^{2}}{1-2 x-\ddots}}}} .
$$

Thus the corresponding numbers $t_{n}$ are given by $t_{0}=2$ and $t_{n}=1$ for $n>0$ which implies $d_{0}(n)=2^{n-1}$ for $n \geq 1$.

Let us also consider the aerated sequence $\left(b_{n}\right)$ with $b_{2 n}=B_{n}$ and $b_{2 n+1}=0$. Here we get 


$$
b(x)=B\left(x^{2}\right)=\frac{1}{1-2 x^{2} C(x)^{2}}=\frac{1}{1-\frac{2 x^{2}}{1-\frac{x^{2}}{1-\frac{x^{2}}{1-\ddots}}} .}
$$

In this case $s_{n}=0, t_{0}=2$, and $t_{n}=1$ for $n>0$. Here we also get $\operatorname{det}\left(b_{i+j}\right)_{i, j=0}^{n-1}=2^{n-1}$ for $n>0$. The corresponding orthogonal polynomials satisfy $p_{0}(x)=1, p_{1}(x)=x$, $p_{2}(x)=x p_{1}(x)-2$ and $p_{n}(x)=x p_{n-1}(x)-p_{n-2}(x)$ for $n>2$. The first terms are $1, x, x^{2}-2, x^{3}-3 x, \ldots$

Now recall that the Lucas polynomials

$$
L_{n}(x)=\sum_{k=0}^{\left\lfloor\frac{n}{2}\right\rfloor}(-1)^{k}\left(\begin{array}{c}
n-k \\
k
\end{array}\right) \frac{n}{n-k} x^{n-2 k}
$$

for $n>0$ satisfy $L_{n}(x)=x L_{n-1}(x)-L_{n-2}(x)$ with initial values $L_{0}(x)=2$ and $L_{1}(x)=x$. The first terms are $2, x, x^{2}-2, x^{3}-3 x, \ldots$ Thus $p_{n}(x)=\bar{L}_{n}(x)$, where $\bar{L}_{n}(x)=L_{n}(x)$ for $n>0$ and $\bar{L}_{0}(x)=1$.

For the numbers $a_{n}(j)$ we get

$$
\begin{aligned}
& a_{2 n}(2 j)=\left(\begin{array}{c}
2 n \\
n-j
\end{array}\right), \\
& a_{2 n+1}(2 j+1)=\left(\begin{array}{c}
2 n+1 \\
n-j
\end{array}\right),
\end{aligned}
$$

and $a_{n}(j)=0$ else. Equivalently $a_{n}(n-2 j)=\left(\begin{array}{c}n \\ j\end{array}\right)$ and $a_{n}(k)=0$ else.

For the proof it suffices to verify (15) which reduces to the trivial identities $\left(\begin{array}{c}2 n \\ n\end{array}\right)=2\left(\begin{array}{c}2 n-1 \\ n-1\end{array}\right),\left(\begin{array}{c}2 n \\ n-j\end{array}\right)=\left(\begin{array}{c}2 n-1 \\ n-j\end{array}\right)+\left(\begin{array}{c}2 n-1 \\ n-1-j\end{array}\right)$, and $\left(\begin{array}{c}2 n+1 \\ n-j\end{array}\right)=\left(\begin{array}{c}2 n \\ n-j\end{array}\right)+\left(\begin{array}{c}2 n \\ n-1-j\end{array}\right)$. Identity (16) reduces to

$$
\sum_{k=0}^{\left\lfloor\frac{n}{2}\right\rfloor}\left(\begin{array}{l}
n \\
k
\end{array}\right) \bar{L}_{n-2 k}=x^{n} \text {. }
$$

\section{Some well-known applications of these methods}

Now let us consider

$$
d_{1}(n)=\operatorname{det}\left(\begin{array}{c}
2 i+2 j+1 \\
i+j
\end{array}\right) .
$$

The generating function of the sequence $\left(\begin{array}{c}2 n+1 \\ n\end{array}\right)$ is 


$$
\sum_{n \geq 0}\left(\begin{array}{c}
2 n+1 \\
n
\end{array}\right) x^{n}=\frac{1}{2} \sum_{n \geq 0}\left(\begin{array}{c}
2 n+2 \\
n+1
\end{array}\right) x^{n}=\frac{1}{2 x}\left(\frac{1}{\sqrt{1-4 x}}-1\right)=\frac{C(x)}{\sqrt{1-4 x}}
$$

Now we have

$$
\begin{aligned}
\sqrt{1-4 x} & =1-2 x C(x)=\left(C(x)-x C(x)^{2}\right)-2 x C(x)=C(x)(1-2 x-x C(x)) \\
& =C(x)\left(1-2 x-x\left(1+x C(x)^{2}\right)\right)=C(x)\left(1-3 x-x^{2} C(x)^{2}\right)
\end{aligned}
$$

Therefore

$$
\frac{C(x)}{\sqrt{1-4 x}}=\frac{1}{1-3 x-x^{2} C(x)^{2}}=\frac{1}{1-3 x-\frac{x^{2}}{1-2 x-\frac{x^{2}}{1-2 x-\frac{x^{2}}{1-2 x-\ddots}}} .}
$$

The corresponding sequences $s_{n}, t_{n}$ are $s_{0}=3, s_{n}=2$ for $n>0$ and $t_{n}=1$. Thus $d_{1}(n)=1$. The corresponding $a_{i}(j)$ are $a_{i}(j)=\left(\begin{array}{c}2 i+1 \\ i-j\end{array}\right)$.

To prove this we must verify (15) which reduces to

$$
\begin{aligned}
& \left(\begin{array}{c}
1 \\
-j
\end{array}\right)=[j=0] \\
& \left(\begin{array}{c}
2 n+1 \\
n
\end{array}\right)=3\left(\begin{array}{c}
2 n-1 \\
n-1
\end{array}\right)+\left(\begin{array}{c}
2 n-1 \\
n-2
\end{array}\right) \\
& \left(\begin{array}{c}
2 n+1 \\
n-j
\end{array}\right)=\left(\begin{array}{c}
2 n-1 \\
n-j
\end{array}\right)+2\left(\begin{array}{c}
2 n-1 \\
n-1-j
\end{array}\right)+\left(\begin{array}{c}
2 n-1 \\
n-2-j
\end{array}\right) .
\end{aligned}
$$

The first line is clear. The right-hand side of the second line gives

$$
\begin{aligned}
3\left(\begin{array}{c}
2 n-1 \\
n-1
\end{array}\right)+\left(\begin{array}{c}
2 n-1 \\
n-2
\end{array}\right) & =2\left(\begin{array}{c}
2 n-1 \\
n-1
\end{array}\right)+\left(\begin{array}{c}
2 n \\
n-1
\end{array}\right) \\
& =\left(\begin{array}{c}
2 n \\
n
\end{array}\right)+\left(\begin{array}{c}
2 n \\
n-1
\end{array}\right)=\left(\begin{array}{c}
2 n+1 \\
n
\end{array}\right) .
\end{aligned}
$$

For the third line we get

$$
\left(\begin{array}{c}
2 n-1 \\
n-j
\end{array}\right)+2\left(\begin{array}{c}
2 n-1 \\
n-1-j
\end{array}\right)+\left(\begin{array}{c}
2 n-1 \\
n-2-j
\end{array}\right)=\left(\begin{array}{c}
2 n \\
n-j
\end{array}\right)+\left(\begin{array}{c}
2 n \\
n-j-1
\end{array}\right)=\left(\begin{array}{c}
2 n+1 \\
n-j
\end{array}\right)
$$

By 17 we see that with

$$
A_{n}=\left(\left(\begin{array}{c}
2 i+1 \\
i-j
\end{array}\right)\right)_{i, j=0}^{n-1}
$$

we get 


$$
A_{n} A_{n}^{\top}=\left(\left(\begin{array}{c}
2 i+2 j+1 \\
i+j
\end{array}\right)\right)_{i, j=0}^{n-1}
$$

Let us give a direct proof of (43). Observe first that

$$
\sum_{l=0}^{n-1}\left(\begin{array}{c}
2 i+1 \\
i-l
\end{array}\right)\left(\begin{array}{c}
2 j+1 \\
j-l
\end{array}\right)=\sum_{l=0}^{i}\left(\begin{array}{c}
2 i+1 \\
i-l
\end{array}\right)\left(\begin{array}{c}
2 j+1 \\
j-l
\end{array}\right)=\sum_{l=0}^{j}\left(\begin{array}{c}
2 i+1 \\
i-l
\end{array}\right)\left(\begin{array}{c}
2 j+1 \\
j-l
\end{array}\right)
$$

and that

$$
\begin{aligned}
\sum_{l=0}^{i}\left(\begin{array}{c}
2 i+1 \\
i-l
\end{array}\right)\left(\begin{array}{c}
2 j+1 \\
j-l
\end{array}\right) & =\sum_{l=0}^{i}\left(\begin{array}{c}
2 i+1 \\
i-l
\end{array}\right)\left(\begin{array}{c}
2 j+1 \\
j+1+l
\end{array}\right) \\
& =\sum_{k=j+1}^{i+j+1}\left(\begin{array}{c}
2 i+1 \\
i+j+1-k
\end{array}\right)\left(\begin{array}{c}
2 j+1 \\
k
\end{array}\right)
\end{aligned}
$$

and

$$
\begin{aligned}
\sum_{l=0}^{j}\left(\begin{array}{c}
2 i+1 \\
i-l
\end{array}\right)\left(\begin{array}{c}
2 j+1 \\
j-l
\end{array}\right) & =\sum_{l=0}^{j}\left(\begin{array}{c}
2 i+1 \\
i+1+l
\end{array}\right)\left(\begin{array}{c}
2 j+1 \\
j-l
\end{array}\right) \\
& =\sum_{k=0}^{j}\left(\begin{array}{c}
2 i+1 \\
i+j+1-k
\end{array}\right)\left(\begin{array}{c}
2 j+1 \\
k
\end{array}\right)
\end{aligned}
$$

Adding the last two equations and using (44) to rewrite the left-hand side, we obtain

$$
\begin{aligned}
& 2 \sum_{l=0}^{n-1}\left(\begin{array}{c}
2 i+1 \\
i-l
\end{array}\right)\left(\begin{array}{c}
2 j+1 \\
j-l
\end{array}\right) \\
& =\sum_{k=0}^{j}\left(\begin{array}{c}
2 i+1 \\
i+j+1-k
\end{array}\right)\left(\begin{array}{c}
2 j+1 \\
k
\end{array}\right)+\sum_{k=j+1}^{i+j+1}\left(\begin{array}{c}
2 i+1 \\
i+j+1-k
\end{array}\right)\left(\begin{array}{c}
2 j+1 \\
k
\end{array}\right) \\
& =\sum_{k=0}^{i+j+1}\left(\begin{array}{c}
2 i+1 \\
i+j+1-k
\end{array}\right)\left(\begin{array}{c}
2 j+1 \\
k
\end{array}\right)=\left(\begin{array}{c}
2 i+2 j+2 \\
i+j+1
\end{array}\right)=2\left(\begin{array}{c}
2 i+2 j+1 \\
i+j
\end{array}\right) .
\end{aligned}
$$

Since $A_{n}$ is a triangle matrix whose diagonal elements are $\left(\begin{array}{c}2 i+1 \\ i-i\end{array}\right)=1$ we get $\operatorname{det}\left(A_{n} A_{n}^{\top}\right)=1$.

\section{A new method}

Fix $k>0$. Let us consider the determinants of the Hankel matrices $B_{n}(k)=$ $\left(\left(\begin{array}{c}2 i+2 j+2 \\ i+j+1-k\end{array}\right)\right)_{i, j=0}^{n-1}$. These have already been computed in [2], Theorem 21. There it 
is shown that

$$
\operatorname{det}\left(B_{k m}(k)\right)=(-1)^{\left(\begin{array}{c}
m \\
2
\end{array}\right) k+m\left(\begin{array}{l}
k \\
2
\end{array}\right)}
$$

and $\operatorname{det}\left(B_{n}(k)\right)=0$ else.

Definition 4.1 Let $\gamma^{(k)}$ be the infinite matrix given by $\gamma_{i j}^{(k)}=1$ if $|i-j|=k$ or $i+j=k-1$ and 0 elsewhere, with rows and columns indexed by $\mathbb{Z}_{\geq 0}$. Let us also consider the finite truncations $\left.\gamma^{(k)}\right|_{N}$, where $\left.A\right|_{N}$ denotes the submatrix consisting of the first $N$ rows and columns of a matrix $A$. We shall also write $\gamma^{(1)}=\gamma$ and $\left.\gamma^{(k)}\right|_{N}=\gamma_{N}^{(k)}$

For example $\gamma_{5}^{(1)}$ and $\gamma_{5}^{(2)}$ are the following matrices:

$$
\gamma_{5}^{(1)}=\left(\begin{array}{lllll}
1 & 1 & 0 & 0 & 0 \\
1 & 0 & 1 & 0 & 0 \\
0 & 1 & 0 & 1 & 0 \\
0 & 0 & 1 & 0 & 1 \\
0 & 0 & 0 & 1 & 0
\end{array}\right) \quad \gamma_{5}^{(2)}=\left(\begin{array}{lllll}
0 & 1 & 1 & 0 & 0 \\
1 & 0 & 0 & 1 & 0 \\
1 & 0 & 0 & 0 & 1 \\
0 & 1 & 0 & 0 & 0 \\
0 & 0 & 1 & 0 & 0
\end{array}\right)
$$

An alternative description will be useful in this section and the next. Let $J_{n}$ be the exchange matrix with 1 's on its antidiagonal and 0's elsewhere. Let $Q_{n}$ be the block matrix $\left(J_{n} I_{n}\right)$. Let $\sigma_{n}$ be the shift matrix with $(i, j)$ entry equal to 1 if $j=i-1$ and 0 otherwise. Then $\gamma_{n}^{(k)}=Q_{n} \sigma_{2 n}^{k} Q_{n}^{\top}$.

\section{Theorem 4.2}

$$
A_{n} \gamma_{n}^{(k)} A_{n}^{\top}=B_{n}(k)
$$

Proof If we set $\gamma_{n}^{(0)}=2 I_{n}$, where $I_{n}$ denotes the $n \times n$-identity matrix, then we already know that (50) holds for $k=0$.

For the general case, we have $\left(A_{n} Q_{n}\right)_{i j}=\left(\begin{array}{c}2 i+1 \\ i+j-(n-1)\end{array}\right)$. Hence the $(i, j)$ entry of $A_{n} Q_{n} \sigma_{2 n} Q_{n}^{\top} A_{n}^{\top}$ is

$$
\begin{aligned}
\sum_{0 \leq r, s \leq n-1}\left(A_{n} Q_{n}\right)_{i r}\left(\sigma^{k}\right)_{r s}\left(A_{n} Q_{n}\right)_{j s} & =\sum_{0 \leq r, s \leq n-1}\left(\begin{array}{c}
2 i+1 \\
i+r-(n-1)
\end{array}\right) \delta_{r-k, s}\left(\begin{array}{c}
2 j+1 \\
j+s-(n-1)
\end{array}\right) \\
& =\sum_{r=n-1-i}^{n+i}\left(\begin{array}{c}
2 i+1 \\
i+r-(n-1)
\end{array}\right)\left(\begin{array}{c}
2 j+1 \\
j+r-k-(n-1)
\end{array}\right) \\
& =\sum_{r^{\prime}=0}^{2 i+1}\left(\begin{array}{c}
2 i+1 \\
r^{\prime}
\end{array}\right)\left(\begin{array}{c}
2 j+1 \\
j-i+r^{\prime}-k
\end{array}\right) \\
& =\left(\begin{array}{c}
2 i+2 j+2 \\
i+j+1-k
\end{array}\right) .
\end{aligned}
$$

The last identity follows from the Chu-Vandermonde formula. 
Lemma 4.3

$$
\begin{aligned}
& \operatorname{det}\left(\gamma_{2 k n}^{(k)}\right)=(-1)^{k n} \\
& \operatorname{det}\left(\gamma_{2 k n+k}^{(k)}\right)=(-1)^{k n+\left(\begin{array}{c}
k \\
2
\end{array}\right)}
\end{aligned}
$$

and all other determinants $\operatorname{det}\left(\gamma_{n}^{(k)}\right)$ vanish.

Proof By the definition of a determinant we have

$$
\operatorname{det}\left(a_{i, j}\right)_{i, j=0}^{n-1}=\sum_{\pi} \operatorname{sgn}(\pi) a_{0, \pi(0)} a_{1, \pi(1)} \cdots a_{n-1, \pi(n-1)}
$$

where $\pi$ runs over all permutations of the set $\{0,1, \ldots, n-1\}$. The determinants of the matrices $\gamma_{n}^{(k)}$ either vanish or the sum over all permutations reduces to a single term $\operatorname{sgn} \pi_{n} c\left(0, \pi_{n}(0), k\right) c\left(1, \pi_{n}(1), k\right) \cdots c\left(n-1, \pi_{n}(n-1), k\right)$.

Let us first consider $k=1$. The last row of $\gamma_{n}^{(1)}$ has only one non-vanishing element $c(n-1, n-2,1)$. Thus each $\pi$ which occurs in the determinant must satisfy $\pi(n-1)=n-2$. The next row from below contains two non-vanishing elements $c(n-2, n-3,1)$ and $c(n-2, n-1,1)$. The last element is the only element of the last column. Therefore we must have $\pi(n-2)=n-1$. The next row from below contains again two non-vanishing elements, $c(n-3, n-4)$ and $c(n-3, n-2)$. But since $n-2$ already occurs as image of $\pi$ we must have $\pi(n-3)=n-4$. Thus the situation has been reduced to $\gamma_{n-2}^{(1)}$. In order to apply induction we need the two initial cases $\gamma_{1}^{(1)}$ and $\gamma_{2}^{(1)}$.

For $n=1$ we get $\pi(0)=0$ and for $n=2 \pi(0)=1$ and $\pi(1)=0$ since

$$
\gamma_{2}^{(1)}=\left(\begin{array}{ll}
1 & 1 \\
1 & 0
\end{array}\right)
$$

If we write $\pi=\pi(0) \cdots \pi(n-1)$ we get in this way $\pi_{1}=0, \pi_{2}=10, \pi_{3}=021$, $\pi_{4}=1032, \ldots$ This gives $\operatorname{sgn} \pi_{n}=-\operatorname{sgn} \pi_{n-2}$ and thus by induction $\operatorname{det} \gamma_{n}^{(1)}=$

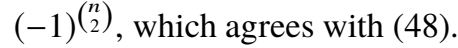

For general $k$ the situation is analogous. The last $k$ rows and columns contain only one non-vanishing element. This implies $\pi(n-j)=n-j-k$ and $\pi(n-j-k)=n-j$ for $1 \leq j \leq k$ and $n \geq 2 k$. Hence $\pi$ restricts to a permutation of $\{0,1, \ldots, n-2 k-1\}$. Thus the determinant can be reduced to $\gamma_{n-2 k}^{(k)}$ and we get $\operatorname{det} \gamma_{n}^{(k)}=(-1)^{k} \operatorname{det} \gamma_{n-2 k}^{(k)}$ if $n \geq 2 k$.

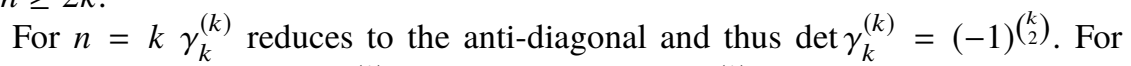
$0<n<k$ the first row of $\gamma_{n}^{(k)}$ vanishes and thus det $\gamma_{n}^{(k)}=0$. For $k<n<2 k$ there are two identical rows because $c(k-1,0, k)=c(k, 0, k)=1$ and $c(k-1, j, k)=$ $c(k, j, k)=0$ for $0<j<n$. Thus we see by induction that 


$$
\begin{aligned}
& \operatorname{det}\left(\gamma_{2 k n}^{(k)}\right)=(-1)^{k n} \\
& \operatorname{det}\left(\gamma_{2 k n+k}^{(k)}\right)=(-1)^{k n+\left(\begin{array}{c}
k \\
2
\end{array}\right)}
\end{aligned}
$$

and all other determinants vanish. This is the same as (48) because $(-1)\left(\begin{array}{c}2 n \\ 2\end{array}\right) k+2 n\left(\begin{array}{c}k \\ 2\end{array}\right)=$

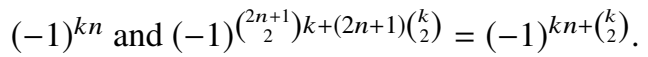

\section{Two useful matrices}

For the finite matrices $\gamma_{N}=\left.\gamma\right|_{N}$ we have $\gamma_{N}^{k} \neq\left.\gamma^{k}\right|_{N}$. In order to compute $\left.\gamma^{k}\right|_{N}$ in the realm of $N$-by- $N$-matrices we introduce the auxiliary matrices $\alpha_{N}^{(k)}$ and $\beta_{N}^{(k)}$.

Let $J_{N}$ be the exchange matrix with 1's on its antidiagonal and 0's elsewhere. Let $Q_{N}$ be the block matrix $\left(J_{N} I_{N}\right)$. Let $\sigma_{N}(\varepsilon)$ be given by

$$
\left(\sigma_{N}(\varepsilon)\right)_{i j}= \begin{cases}1 & \text { if } i=j+1 \\ \varepsilon & \text { if }(i, j)=(0, N-1) \\ 0 & \text { otherwise }\end{cases}
$$

Define $\alpha_{N}^{(k)}$ and $\beta_{N}^{(k)}$ as

$$
Q_{N} \sigma_{2 N}(\varepsilon)^{k} Q_{N}^{\top}= \begin{cases}\alpha_{N}^{(k)} & \text { if } \varepsilon=1 \\ \beta_{N}^{(k)} & \text { if } \varepsilon=-1 \\ \gamma_{N}^{(k)} & \text { if } \varepsilon=0\end{cases}
$$

and the last line has been stated before. We shall again suppress the superscripts when $k=1$.

As a slight variation, consider the following infinite square matrices with rows and columns indexed by $\mathbb{Z} \backslash\{0\}=\{\ldots,-2,-1,1,2, \ldots\}$. Let $\bar{I}$ be the identity matrix and let $\bar{J}$ be the exchange matrix with $\bar{J}_{n,-n}=1$ for all $n$ and 0 elsewhere. Let $\bar{\sigma}$ be given by $\bar{\sigma}_{n, n-1}=1$ and 0 elsewhere. Define also the infinite rectangular matrix $\bar{Q}$ with rows indexed by $\mathbb{Z}_{+}=\{1,2, \ldots\}$ and columns indexed by $\mathbb{Z} \backslash\{0\}$ by $\bar{Q}_{|n|, n}=1$ for $n \in \mathbb{Z} \backslash\{0\}$ and 0 elsewhere. Note that $\gamma^{(k)}=\bar{Q} \bar{\sigma}^{k} \bar{Q}^{T}$, after shifting indices from $\mathbb{Z}_{\geq 0}$ to $\mathbb{Z}_{+}$.

Theorem 5.1 When $\delta$ stands for either $\alpha_{N}, \beta_{N}$, or $\gamma$ one has $\delta^{(k)}=\delta \cdot \delta^{(k-1)}-\delta^{(k-2)}$ with initial values $\delta^{(1)}=\delta$ and $\delta^{(0)}=2$.

Proof For $\alpha_{N}$ and $\beta_{N}$, take $\sigma=\sigma_{2 N}( \pm 1), Q=Q_{N}, J=J_{2 N}$, and $I=I_{2 N}$. For $\gamma$, take $\sigma=\bar{\sigma}, Q=\bar{Q}, J=\bar{J}$, and $I=\bar{I}$. Note that in either case $Q^{\top} Q=I+J$, $\sigma J \sigma=J$, and $Q J=Q$. For $k \geq 2$, 


$$
\begin{aligned}
\delta \cdot \delta^{(k-1)} & =Q \sigma Q^{\top} Q \sigma^{k-1} Q^{\top} \\
& =Q \sigma(I+J) \sigma^{k-1} Q^{\top} \\
& =Q \sigma^{k} Q^{\top}+Q(\sigma J \sigma) \sigma^{k-2} Q^{\top} \\
& =\delta^{(k)}+\delta^{(k-2)} .
\end{aligned}
$$

By induction we see that each $\gamma^{(k)}$ is a polynomial in $\gamma$. Therefore all $\gamma^{(k)}$ commute. Theorem 5.1 shows that the matrices $\gamma^{(k)}$ are Lucas polynomials in $\gamma$. More precisely

$$
\gamma^{(k)}=L_{k}(\gamma) .
$$

By the same argument, $\alpha_{N}^{(k)}=L_{k}\left(\alpha_{N}\right)$ and $\beta_{N}^{(k)}=L_{k}\left(\beta_{N}\right)$.

Theorem 5.2 For any polynomial $p$ with $\operatorname{deg} p \leq 2 N$, $\frac{p\left(\alpha_{N}\right)+p\left(\beta_{N}\right)}{2}=\left.p(\gamma)\right|_{N}$.

Proof Note that $\left(L_{0}, \ldots, L_{2 N}\right)$ is a basis of the vector space of degree at most $2 N$ polynomials, since $\operatorname{deg}\left(L_{k}\right)=k$. Therefore it suffices to show that $\left(L_{k}\left(\alpha_{N}\right)+\right.$ $\left.L_{k}\left(\beta_{N}\right)\right) / 2=\left.L_{k}(\gamma)\right|_{N}$ for $k \leq 2 N$. To wit,

$$
\begin{aligned}
\left(L_{k}\left(\alpha_{N}\right)+L_{k}\left(\beta_{N}\right)\right) / 2 & =\left(\alpha_{N}^{(k)}+\beta_{N}^{(k)}\right) / 2 \\
& =Q_{N}\left(\sigma_{2 N}(1)^{k}+\sigma_{2 N}(-1)^{k}\right) Q_{N}^{\top} / 2 \\
& =Q_{N} \sigma_{2 N}(0)^{k} Q_{N}^{\top} \\
& =\gamma_{N}^{(k)} \\
& =\left.L_{k}(\gamma)\right|_{N} .
\end{aligned}
$$

\section{Relating the determinant to the $\gamma$ matrices}

Let $a_{n}, b_{n}$, and $g_{n}$ be the characteristic polynomials of $\alpha_{n}, \beta_{n}$, and $\gamma_{n}$, respectively. By cofactor expansion along the last row we get $g_{n}(x)=x g_{n-1}(x)-g_{n-2}(x), a_{n}(x)=$ $g_{n}(x)-g_{n-1}(x)$, and $b_{n}(x)=g_{n}(x)+g_{n-1}(x)$. This plus the initial conditions of the $n=1$ and 2 cases gives $b_{n}(x)=L_{n}(x)$,

$$
g_{n}(x)=\sum_{k=0}^{n}(-1)^{n-k} \bar{L}_{k}(x)
$$

and

$$
a_{n}(x)=L_{n}(x)+2 \sum_{k=0}^{n-1}(-1)^{n-k} \bar{L}_{k}(x) .
$$

Here $\bar{L}_{n}(x)$ is the Lucas polynomial $L_{n}(x)$ except when $n=0$, in which case it is 1 . 
By Theorem 4.2, $A \phi(\gamma) A^{\top}$ is Hankel for all polynomials $\phi$. Here $A$ represents the infinite matrix $\left(\left(\begin{array}{c}2 i+1 \\ i-j\end{array}\right)\right)_{i, j \geq 0}$ with finite truncations $\left.A\right|_{n}=A_{n}$. This is because $\phi(x)$ can be expanded as a sum of Lucas polynomials $L_{k}(x)$, each of which gives a Hankel matrix. Moreover, multiplying the polynomial by $(x+2)$ shifts the Hankel matrix forward by 1 . It suffices to show this for $L_{k}(x)$. Recall that

$$
A L_{k}(\gamma) A^{\top}=A \gamma^{(k)} A^{\top}=\left(\left(\begin{array}{c}
2 i+2 j+2 \\
i+j+1-k
\end{array}\right)\right)_{i, j \geq 0}
$$

Then by Theorem 5.1

$$
A L_{k}(\gamma)(\gamma+2) A^{\top}=A\left(\gamma^{(k-1)}+\gamma^{(k+1)}+2 \gamma^{(k)}\right) A^{\top}
$$

has $(i, j)$ entry

$$
\left(\begin{array}{c}
2 i+2 j+2 \\
i+j+2-k
\end{array}\right)+2\left(\begin{array}{c}
2 i+2 j+2 \\
i+j+1-k
\end{array}\right)+\left(\begin{array}{c}
2 i+2 j+2 \\
i+j-k
\end{array}\right)=\left(\begin{array}{c}
2 i+2 j+4 \\
i+j+2-k
\end{array}\right)
$$

by Pascal's identity, which is the $(i+1, j)$ entry of the original matrix.

We'll now write the Hankel matrices of the sequence $\left(\left(\begin{array}{c}2 n+r \\ n\end{array}\right)\right)_{n \geq 0}$ in terms of the $\gamma$ matrices and $A$. By the above results, when $i+j=n$ we have

$$
\left(A b_{k}(\gamma) A^{\top}\right)_{i j}=\left(A L_{k}(\gamma) A^{\top}\right)_{i j}=\left(\begin{array}{c}
2 n+2 \\
n+1-k
\end{array}\right)
$$

and

$$
\left(A b_{k}(\gamma)(\gamma+2)^{k-1} A^{\top}\right)_{i j}=\left(\begin{array}{c}
2(n+k-1)+2 \\
(n+k-1)+1-k
\end{array}\right)=\left(\begin{array}{c}
2 n+2 k \\
n
\end{array}\right) .
$$

By induction on $k$, we'll show $\left(A g_{k}(\gamma) A^{\top}\right)_{i j}=\left(\begin{array}{c}2 n+1 \\ n-k\end{array}\right)$. The $k=0$ case is (43). For $k \geq 1$,

$$
\begin{aligned}
\left(A g_{k}(\gamma) A^{\top}\right)_{i j} & =\left(A\left(b_{k}(\gamma)-g_{k-1}(\gamma)\right) A^{\top}\right)_{i j} \\
& =\left(\begin{array}{c}
2 n+2 \\
n+1-k
\end{array}\right)-\left(\begin{array}{c}
2 n+1 \\
n-(k-1)
\end{array}\right)=\left(\begin{array}{c}
2 n+1 \\
n-k
\end{array}\right) .
\end{aligned}
$$

Hence

$$
\left(A g_{k}(\gamma)(\gamma+2)^{k} A^{\top}\right)_{i j}=\left(\begin{array}{c}
2(n+k)+1 \\
(n+k)-k
\end{array}\right)=\left(\begin{array}{c}
2 n+2 k+1 \\
n
\end{array}\right)
$$

We've proven the following theorem.

Theorem 6.1 For $r \geq 1$, let $k=\left\lfloor\frac{r}{2}\right\rfloor$ and $l=\left\lfloor\frac{r-1}{2}\right\rfloor$, and define the function

$$
h_{r}(x)= \begin{cases}g_{k}(x) & \text { if } r=2 k+1 \\ b_{k}(x) & \text { if } r=2 k\end{cases}
$$


For $N \geq k+l$, by Theorem 5.2, $d_{r}(N)$ equals

$$
\operatorname{det}\left(\left.h_{r}(\gamma)(\gamma+2)^{l}\right|_{N}\right)=\operatorname{det}\left(\frac{1}{2}\left(h_{r}\left(\alpha_{N}\right)\left(\alpha_{N}+2\right)^{l}+h_{r}\left(\beta_{N}\right)\left(\beta_{N}+2\right)^{l}\right)\right) .
$$

\section{Structure of the matrices}

In this section we determine the structure of the matrices $\left(\beta_{N}+2\right)^{-1}, g_{k}\left(\alpha_{N}\right), g_{k}\left(\beta_{N}\right)$, $b_{k}\left(\alpha_{N}\right)$, and $b_{k}\left(\beta_{N}\right)$, as well as the determinants of $\left.g_{k}(\gamma)\right|_{N}$ and $\left.b_{k}(\gamma)\right|_{N}$.

To determine $p\left(\alpha_{N}\right)$ and $p\left(\beta_{N}\right)$ for a polynomial $p$ of degree less than $N$, we begin by writing $p(\gamma)$ as a sum of $\gamma^{(k)}$ matrices using the multiplicative formula of Theorem 5.1. We then apply Prop 7.2 to show that $p\left(\alpha_{N}\right)$ and $p\left(\beta_{N}\right)$ are the same as $\left.p(\gamma)\right|_{N}$ on and above the anti-diagonal. The structure of $p\left(\alpha_{N}\right)$ follows from the symmetry of $\alpha_{N}$ across its anti-diagonal. The structure of $p\left(\beta_{N}\right)$ can be computed from $p\left(\alpha_{N}\right)$ and $\left.p(\gamma)\right|_{N}$ with Theorem 5.2 .

Proposition 7.1 The determinant of a block matrix

$$
\left(\begin{array}{ll}
A & B \\
C & D
\end{array}\right)
$$

where $A$ and $D$ are square and $D$ is invertible is $\operatorname{det}(D) \operatorname{det}\left(A-B D^{-1} C\right)$.

Proof Note that

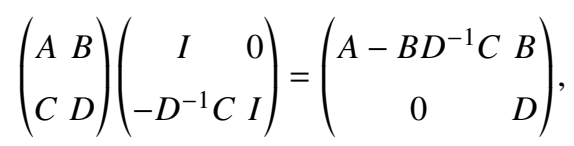

and that the determinant of a block-triangular matrix is the product of the determinants of its diagonal blocks.

Proposition 7.2 Let $T$ be a $N$-by- $N$ tridiagonal matrix and let $p$ be a polynomial of degree $d$. Let $v$ be the $N$-by-1 column vector with a 1 in its last entry and 0 elsewhere. Then the $(i, j)$ entries of $p(T)$ and $p\left(T+v v^{\top}\right)$ agree when $i+j \leq 2(N-1)-d$.

Proof It suffices to prove this for $p(x)=x^{d}$. Call a $N$-by- $N$ matrix " $k$-small" iff its entries $(i, j)$ with $i+j \leq 2(N-1)-k$ are all 0 . For instance, $v v^{\top}$ is 1 -small.

Suppose a matrix $M$ is $k$-small. For $i+j \leq 2(N-1)-k-1$, the $(i, j)$ entry of $T M$ is $\sum_{l=0}^{N-1} T_{i l} M_{l j}=T_{i, i-1} M_{i-1, j}+T_{i, i} M_{i, j}+T_{i, i+1} M_{i+1, j}$. Since $M$ is $k$-small, its $(i-1, j),(i, j)$, and $(i+1, j)$ entries are 0 , which implies that $T M$ is $(k+1)$-small. Similarly, $M T, v v^{\top} M$, and $M v v^{\top}$ are $(k+1)$-small.

Consider $\left(T+v v^{\top}\right)^{d}-T^{d}$. Expanding the binomial product yields $2^{d}-1$ terms, all of which are products of $d T^{\prime}$ 's and $v v^{\top}$ 's and contain at least one $v v^{\top}$. It follows from the above that each of these terms is $d$-small, so $p\left(T+v v^{\top}\right)-p(T)$ is $d$-small. 
Lemma 7.3 The inverse of $\left(\beta_{N}+2\right)$ is $\left(\frac{1}{2}(-1)^{i+j}(2 \min \{i, j\}+1)\right)_{i, j=0}^{N-1}$. The determinant of $\left(\beta_{N}+2\right)$ is 2 . For example,

$$
\left(\beta_{5}+2\right)^{-1}=\frac{1}{2}\left(\begin{array}{rrrrr}
1 & -1 & 1 & -1 & 1 \\
-1 & 3 & -3 & 3 & -3 \\
1 & -3 & 5 & -5 & 5 \\
-1 & 3 & -5 & 7 & -7 \\
1 & -3 & 5 & -7 & 9
\end{array}\right) .
$$

Proof For $i \neq 0, N-1$ the row $i$ of $\left(\beta_{N}+2\right)$ is $\left(2 \delta_{i l}+\delta_{i, l-1}+\delta_{i, l+1}\right)_{l=0}^{N-1}$. The product of this with column $j$ of the claimed inverse is

$$
\begin{aligned}
& \sum_{l=0}^{N-1}\left(2 \delta_{i l}+\delta_{i, l-1}+\delta_{i, l+1}\right) \frac{1}{2}(-1)^{l+j}(2 \min \{l, j\}+1) \\
& =\frac{1}{2}(-1)^{i+j}(4 \min \{i, j\}+2-2 \min \{i+1, j\}-1-2 \min \{i-1, j\}-1) \\
& =(-1)^{i+j}(2 \min \{i, j\}-\min \{i+1, j\}-\min \{i-1, j\}) .
\end{aligned}
$$

This is 0 if $i+1 \leq j$ or $i-1 \geq j$ and is 1 if $i=j$.

The first row of $\left(\beta_{N}+2\right)$ is $(3,1,0, \ldots, 0)$, and the last row is $(0, \ldots, 0,1,1)$. Column $j \neq 0, N-1$ of the claimed inverse begins and ends as

$$
\frac{1}{2}\left((-1)^{j},(-1)^{j+1} 3, \ldots,(-1)^{j+N-2}(2 j+1),(-1)^{j+N-1}(2 j+1)\right),
$$

so it kills the first and last rows of $\left(\beta_{N}+2\right)$. Column 0 of the claimed inverse begins and ends as $\frac{1}{2}\left(1,-1, \ldots,(-1)^{N-2},(-1)^{N-1}\right)$ while column $N-1$ begins and ends as $\frac{1}{2}\left((-1)^{N-1},(-1)^{N} 3, \ldots,-(2 N-3), 2 N-1\right)$. It's easy to verify that these columns have the correct products with rows of $\left(\beta_{N}+2\right)$.

The determinant $\operatorname{det}(\beta+2)$ is $(-1)^{N} b_{N}(-2)$, which can be computed with recurrence in Section 6 to be 2 .

Lemma 7.4 For $k<N$, the $(i, j)$ entry of $g_{k}\left(\alpha_{N}\right)$ is $(-1)^{i+j+k}$ if $k \leq i+j \leq 2 N-k-2$ and $|i-j| \leq k$ and is 0 otherwise. The $(i, j)$ entry of $g_{k}\left(\beta_{N}\right)$ is $(-1)^{i+j+k}$ if $k \leq i+j \leq 2 N-k-2$ and $|i-j| \leq k$, is $2(-1)^{i+j+k}$ if $2 N-k-1 \leq i+j$, and is 0 otherwise. For example, 


$$
g_{2}\left(\beta_{6}\right)=\left(\begin{array}{rrrrrr}
0 & 0 & 1 & 0 & 0 & 0 \\
0 & 1 & -1 & 1 & 0 & 0 \\
1 & -1 & 1 & -1 & 1 & 0 \\
0 & 1 & -1 & 1 & -1 & 1 \\
0 & 0 & 1 & -1 & 1 & -2 \\
0 & 0 & 0 & 1 & -2 & 2
\end{array}\right) .
$$

Proof Recall that $g_{j}(\gamma)=\gamma^{(j)}-\gamma^{(j-1)}+\cdots \pm \gamma^{(1)} \mp 1$, by (63). Therefore $\frac{1}{2}\left(g_{k}\left(\alpha_{N}\right)+\right.$ $\left.g_{k}\left(\beta_{N}\right)\right)=\left.g_{k}(\gamma)\right|_{N}=\gamma_{N}^{(k)}-\gamma_{N}^{(k-1)}+\cdots \pm \gamma_{N}^{(1)} \mp 1$. From the definition of the $\gamma_{N}^{(j)}$, the $(i, j)$ entry of $\left.g_{k}(\gamma)\right|_{N}$ is $(-1)^{i+j+k}$ if $k \leq i+j$ and $|i-j| \leq k$ and is 0 otherwise.

Note that polynomials in $\alpha_{N}$ are symmetric about their anti-diagonal. Since the degree of $g_{k}$ is $k<N$, Prop 7.2 says that $g_{k}\left(\alpha_{N}\right)$ agrees with $\left.g_{k}(\gamma)\right|_{N}$ on and above its anti-diagonal. Thus, the $(i, j)$ entry of $g_{k}\left(\alpha_{N}\right)$ is $(-1)^{i+j+k}$ if $k \leq i+j \leq 2 N-k-2$ and $|i-j| \leq k$ and is 0 otherwise. Similarly, the $(i, j)$ entry of $g_{k}\left(\beta_{N}\right)=\left.2 g_{k}(\gamma)\right|_{N}-g_{k}\left(\alpha_{N}\right)$ is $(-1)^{i+j+k}$ if $k \leq i+j \leq 2 N-k-2$ and $|i-j| \leq k, 2(-1)^{i+j+k}$ if $2 N-k-1 \leq i+j$, and 0 otherwise.

Lemma 7.5

$$
\left.\operatorname{det} g_{k}(\gamma)\right|_{N}= \begin{cases}1 & \text { if } N=(2 k+1) n \\
(-1)^{\left(\begin{array}{c}
(+1 \\
2
\end{array}\right)} & \text { if } N=(2 k+1) n+k+1 \\
0 & \text { otherwise. }\end{cases}
$$

Proof When $N=0$ the determinant is vacuously 1 . When $0<N<k+1$, the first column is 0 . When $N=k+1$ the matrix is 0 above its antidiagonal and 1 on its

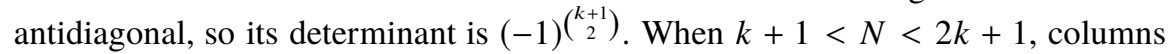
$k-1$ and $k+1$ are equal. Thus the claim holds for all $N<2 k+1$. We'll show that for $N \geq 2 k+1,\left.\operatorname{det} g_{k}(\gamma)\right|_{N}=\left.\operatorname{det} g_{k}(\gamma)\right|_{N-2 k-1}$.

Fix $N \geq 2 k+1$ and let $M=\left.g_{k}(\gamma)\right|_{N}$. Subdivide $M$ into a block matrix consisting of the leading principal order- $N-1$ submatrix $M_{11}$, the bottom-right entry $M_{22}$, and the remainders of the last column and row $M_{12}$ and $M_{21}$. The determinant of $M$ is $\operatorname{det}\left(M_{22}\right) \operatorname{det}\left(M^{\prime}\right)$, where $M^{\prime}$ is the $N-1$-by- $N-1$ matrix $M_{11}-M_{12} M_{22}^{-1} M_{21}$ by Proposition 7.1.

We will perform cofactor expansion in the bottom right of $M^{\prime}$. Since $M_{22}=(-1)^{k}$, the bottom right $k$-by- $k$ submatrix of $M^{\prime}$ is the zero matrix. As a result, the only entry in the bottom row of $M^{\prime}$ is the 1 at $(N-2, N-k-2)$. After deleting its row and column, the only entry in the bottom row of $M^{\prime}$ is the 1 at $(N-3, N-k-3)$. This pattern continues up to the 1 at $(N-k-1, N-2 k-1)$. Since $M^{\prime}$ is symmetric, a similar sequence of lone 1's can be removed in the last $k$ columns.

After the last $2 k$ rows and columns have been removed, $M^{\prime}$ has been reduced to $\left.g_{k}(\gamma)\right|_{N-2 k-1}$. The $2 k$ removed 1's contribute a factor of $(-1)^{k}$ to the determinant, 
which comes from the parity of the permutation $(0 k)(1 k+1) \cdots(k-12 k)$. This cancels with the sign of $M_{22}$.

Lemma 7.6 For $k<N$, the $(i, j)$ entry of $b_{k}\left(\alpha_{N}\right)$ is 1 if $|i-j|=k, i+j=k-1$, or $i+j=2(N-1)-(k-1)$ and is 0 otherwise. The $(i, j)$ entry of $b_{k}\left(\beta_{N}\right)$ is 1 if $|i-j|=k$ or $i+j=k-1$, is -1 if $i+j=2(N-1)-(k-1)$, and is 0 otherwise. In particular $b_{k}(\gamma)=\gamma^{(k)}$. Moreover,

$$
\left.\operatorname{det} b_{k}(\gamma)\right|_{N}= \begin{cases}(-1)^{k n} & \text { if } N=2 k n \\
(-1)^{k n+\left(\begin{array}{c}
k \\
2
\end{array}\right)} & \text { if } N=2 k n+k \\
0 & \text { otherwise. }\end{cases}
$$

Proof The first set of claims follow from the Lemma 7.4 and the fact that $b_{k}(x)=$ $g_{k}(x)+g_{k-1}(x)$. The determinant of $\gamma^{(k)}$ was calculated in Lemma 4.3.

\section{Calculation of the determinant}

In this section we prove the seven formulas mentioned in the introduction. Recall Theorem 6.1 and its notation.

Let $\mu_{i}=\frac{1}{2}\left(\left(\alpha_{N}+2\right)^{i} h_{r}\left(\alpha_{N}\right)+\left(\beta_{N}+2\right)^{i} h_{r}\left(\beta_{N}\right)\right)$ for $0 \leq i \leq l$. From here on we'll suppress the subscripts on $\alpha_{N}$ and $\beta_{N}$. By Theorem 6.1, we're interested in calculating $d_{r}(N)=\operatorname{det} \mu_{l}$. Note that

$$
\mu_{i+1}=\mu_{i}(\beta+2)+(\alpha+2)^{i} h_{r}(\alpha) v v^{\top} .
$$

The results of the previous section give us control over $\mu_{0}$. We will induct on the above equation to screw the smoothing operators $\alpha+2$ and $\beta+2$ into place, using the matrix determinant lemma to keep track of the determinants. In the seven cases proven here, the determinant or adjugate of $\mu_{i}$ is multiplied by a constant factor at each step.

Proposition 8.1 (Matrix determinant lemma) If $A$ is an $n$-by- $n$ matrix and $u$ and $v$ are $n$-by- 1 column vectors, then

$$
\operatorname{det}\left(A+u v^{\top}\right)=\operatorname{det}(A)+v^{\top} \operatorname{adj}(A) u .
$$

Proof This is a polynomial identity in the entries of $A, u$, and $v$, so it suffices to prove it for the dense subset where $A$ is invertible. Consider

$$
\left(\begin{array}{cc}
I & 0 \\
v^{\top} & 1
\end{array}\right)\left(\begin{array}{cc}
I+A^{-1} u v^{\top} & u \\
0 & 1
\end{array}\right)\left(\begin{array}{cc}
I & 0 \\
-v^{\top} & 1
\end{array}\right)=\left(\begin{array}{lc}
I & u \\
0 & 1+v^{\top} A^{-1} u
\end{array}\right),
$$

which shows that $1 \cdot \operatorname{det}\left(I+A^{-1} u v^{\top}\right) \cdot 1=\operatorname{det}\left(1+v^{\top} A^{-1} u\right)$. Multiplying through by $\operatorname{det} A$ yields $\operatorname{det}\left(A+u v^{\top}\right)=\operatorname{det}(A)\left(1+v^{\top} A^{-1} u\right)=\operatorname{det}(A)+v^{\top} \operatorname{adj}(A) u$. 


\subsection{The case that $\mu_{0}$ is invertible}

Lemma 8.2 Suppose there is an $N$-dimensional column vector $w$ such that $\mu_{0} w=$ $h_{r}\left(\alpha_{N}\right) v$ and that the last $l-1$ entries of $h_{r}\left(\beta_{N}\right) w$ are 0 . Then

$$
\operatorname{det}\left(\mu_{l}\right)=\operatorname{det}\left(\mu_{0}\right) 2^{l}\left(1+v^{\top}\left(\beta_{N}+2\right)^{-1} w\right)^{l} .
$$

Proof By Prop 7.2, $(\alpha+2)^{i}$ and $(\beta+2)^{i}$ differ only in the last $i$ columns. It follows from the second hypothesis that $(\beta+2)^{i} h_{r}(\beta) w=(\alpha+2)^{i} h_{r}(\beta) w$ for $0 \leq i<l$. Thus

$$
\mu_{i} w=(\alpha+2)^{i} h_{r}(\alpha) v
$$

and

$$
\operatorname{det}\left(\mu_{i}\right) w=\operatorname{adj}\left(\mu_{i}\right)(\alpha+2)^{i} h_{r}(\alpha) v
$$

for $0 \leq i<l$. By 82 and the matrix determinant lemma,

$$
\begin{aligned}
\operatorname{det}\left(\mu_{i+1}\right) & =\operatorname{det}(\beta+2)\left(\operatorname{det}\left(\mu_{i}\right)+v^{\top}(\beta+2)^{-1} \operatorname{adj}\left(\mu_{i}\right)(\alpha+2)^{i} h_{r}(\alpha) v\right) \\
& =\operatorname{det}(\beta+2)\left(\operatorname{det}\left(\mu_{i}\right)+v^{\top}(\beta+2)^{-1} \operatorname{det}\left(\mu_{i}\right) w\right)
\end{aligned}
$$

Hence

$$
\operatorname{det}\left(\mu_{i+1}\right)=2 \operatorname{det}\left(\mu_{i}\right)\left(1+v^{\top}\left(\beta_{N}+2\right)^{-1} w\right)
$$

Theorem 8.3

$$
\begin{aligned}
& d_{2 k+1}((2 k+1) n)=(2 n+1)^{k} \\
& d_{2 k+1}((2 k+1) n+k+1)=(-1)^{\left(\begin{array}{c}
k+1 \\
2
\end{array}\right)} 4^{k}(n+1)^{k} \\
& d_{2 k}(2 k n)=(-1)^{k n} \\
& d_{2 k}(2 k n+k)=(-1)^{k n+\left(\begin{array}{c}
k \\
2
\end{array}\right) 4^{k-1}(n+1)^{k-1}}
\end{aligned}
$$

Proof Given $w$, it is straightforward to verify the hypotheses and evaluate the final expression of Lemma 8.2 with the lemmas of Section 7 . For the first formula, take $w$ to be the $(2 k+1) n$-dimensional column vector

$$
w_{1}=(-1)^{n-1}\left(\sum_{m=0}^{n-1}(-1)^{m} e_{(2 k+1) m}-\sum_{m=0}^{n-1}(-1)^{m} e_{(2 k+1) m+2 k}\right)+e_{N-1},
$$

where $\left\{e_{i}\right\}_{i=0}^{N-1}$ is the standard basis. Then $g_{k}(\alpha) w_{1}=g_{k}(\beta) w_{1}=e_{N-k-1}$.

For the second formula, take $w$ to be the $(2 k+1) n+k+1$-dimensional column vector

$$
w_{2}=(-1)^{n}\left(\sum_{m=0}^{n}(-1)^{m} e_{(2 k+1) m+k-1}-\sum_{m=0}^{n-1}(-1)^{m} e_{(2 k+1) m+k+1}\right)+e_{N-1},
$$


which gives $g_{k}(\alpha) w_{2}=e_{N-k-1}+e_{N-k}$ and $g_{k}(\beta) w_{2}=e_{N-k-1}-e_{N-k}$.

For the third formula, take $w$ to be the $2 k n$-dimensional column vector

$$
w_{3}=(-1)^{n-1}\left(\sum_{m=0}^{n-1}(-1)^{m} e_{2 k m}-\sum_{m=0}^{n-1}(-1)^{m} e_{2 k m+2 k-1}\right)+e_{N-1} \text {, }
$$

which gives $b_{k}(\alpha) w_{3}=b_{k}(\beta) w_{3}=e_{N-k-1}+e_{N-k}$.

For the fourth formula, take $w$ to be the $2 k n+k$-dimensional column vector

$$
w_{4}=(-1)^{n}\left(\sum_{m=0}^{n}(-1)^{m} e_{2 k m+k-1}-\sum_{m=0}^{n-1}(-1)^{m} e_{2 k m+k+1}\right)+e_{N-1},
$$

which gives $b_{k}(\alpha) w_{4}=e_{N-k-1}+3 e_{N-k}$ and $b_{k}(\beta) w_{4}=e_{N-k-1}-e_{N-k}$.

\subsection{The case that $\mu_{0}$ is singular}

We will make use of the following fact about the adjugate matrix.

Proposition 8.4 The rank of the adjugate $\operatorname{adj}(M)$ of an $n$-by- $n$ matrix $M$ satisfies

$$
\operatorname{rk} \operatorname{adj}(M)= \begin{cases}n & \text { if } \operatorname{rk} M=n \\ 1 & \text { if } \operatorname{rk} M=n-1 \\ 0 & \text { otherwise }\end{cases}
$$

Proof Recall that $\operatorname{adj}(M) \cdot M=\operatorname{det}(M) I$. If $\operatorname{rk} M=n$ then $M$ is invertible with inverse $\frac{1}{\operatorname{det}(M)} \operatorname{adj}(M)$, which also has rank $n$.

If $\operatorname{rk} M=n-1$, then $\operatorname{det}(M)=0$, in which case $\operatorname{adj}(M)$ must send all vectors into the kernel of $M$, which has rank 1 . In this case $M$ also has a nonzero order- $n-1$ $\operatorname{minor}, \operatorname{so} \operatorname{adj}(M)$ has rank 1 .

If $\operatorname{rk} M \leq n-2$, then all order- $n-1$ minors of $M$ are zero, $\operatorname{so} \operatorname{adj}(M)=0$.

Lemma 8.5 Suppose there is a nonzero $N$-dimensional column vector $w$ such that $\operatorname{det}\left(\mu_{0}\right)=0, \operatorname{det}\left(\left.\mu_{0}\right|_{N-1}\right) \neq 0, \mu_{0} w=0, v^{\top} w=1, v^{\top}(\beta+2)^{-1} w \neq 0$, and entries $N-k-l$ through $N-3$ of $w$ are 0 . Then

$$
\operatorname{det}\left(\mu_{l}\right)=\operatorname{det}\left(\left.\mu_{0}\right|_{N-1}\right)\left(2 v^{\top}\left(\beta_{N}+2\right)^{-1} w\right)^{l}\left(w^{\top}(\alpha+2)^{l-1} h_{r}(\alpha) v\right) \text {. }
$$

Proof Let $c=\operatorname{det}\left(\left.\mu_{0}\right|_{N-1}\right)$. We will show by induction that

$$
\operatorname{adj}\left(\mu_{i}\right)=c\left(2 v^{\top}\left(\beta_{N}+2\right)^{-1} w\right)^{i} w w^{\top},
$$

for $0 \leq i<l$. For the base case of $i=0$, note that the first two hypotheses imply that $\mu_{0}$ has rank $N-1$. Since $w$ generates the kernel and $\mu_{0}$ is symmetric, Proposition 
8.4 implies that $\operatorname{adj}\left(\mu_{0}\right)$ is a constant $d$ times $w w^{\top}$. In fact $c=v^{\top} \operatorname{adj}\left(\mu_{0}\right) v=$ $d v^{\top} w w^{\top} v=d$.

Suppose the claim holds for $i$. Since $\alpha+2$ is tridiagonal, the last hypothesis combined with Lemmas 7.4 and 7.6 imply that $w^{\top}(\alpha+2)^{i} h_{r}(\alpha) v=0$. By (82) and the matrix determinant lemma,

$$
\begin{aligned}
\operatorname{det}\left(\mu_{i+1}\right) & =\operatorname{det}(\beta+2)\left(\operatorname{det}\left(\mu_{i}\right)+v^{\top}(\beta+2)^{-1} \operatorname{adj}\left(\mu_{i}\right)(\alpha+2)^{i} h_{r}(\alpha) v\right) \\
& =\operatorname{det}(\beta+2)\left(0+c\left(2 v^{\top}\left(\beta_{N}+2\right)^{-1} w\right)^{i} v^{\top}(\beta+2)^{-1} w w^{\top}(\alpha+2)^{i} h_{r}(\alpha) v\right) \\
& =0
\end{aligned}
$$

so $\mu_{i+1}$ has rank at most $n-1$. Since $(\alpha+2)^{i} h_{r}(\alpha) v v^{\top}$ doesn't affect the bottom-right cofactor,

$$
\begin{aligned}
v^{\top} \operatorname{adj}\left(\mu_{i+1}\right) v & =v^{\top} \operatorname{adj}\left(\mu_{i}(\beta+2)+(\alpha+2)^{i} h_{r}(\alpha) v v^{\top}\right) v \\
& =v^{\top} \operatorname{adj}\left(\mu_{i}(\beta+2)\right) v \\
& =c \operatorname{det}(\beta+2) v^{\top}(\beta+2)^{-1}\left(2 v^{\top}\left(\beta_{N}+2\right)^{-1} w\right)^{i} w w^{\top} v \\
& =c\left(2 v^{\top}\left(\beta_{N}+2\right)^{-1} w\right)^{i+1} .
\end{aligned}
$$

This is nonzero by assumption, $\operatorname{so} \operatorname{adj}\left(\mu_{i+1}\right)$ is nonzero. By Prop 8.4 it is rank 1 . The matrix $\mu_{i+1}$ is symmetric and $w$ lies in its kernel:

$$
w^{\top} \mu_{i+1}=w^{\top} \mu_{i}(\beta+2)+w^{\top}(\alpha+2)^{i} h_{r}(\alpha) v v^{\top}=0+0,
$$

so it is of the form $\operatorname{adj}\left(\mu_{i+1}\right)=c\left(2 v^{\top}\left(\beta_{N}+2\right)^{-1} w\right)^{i+1} w w^{\top}$. This completes the induction.

The final $\mu_{l}$ has determinant

$$
\begin{aligned}
\operatorname{det}\left(\mu_{l}\right) & =\operatorname{det}(\beta+2)\left(\operatorname{det}\left(\mu_{l-1}\right)+v^{\top}(\beta+2)^{-1} \operatorname{adj}\left(\mu_{l-1}\right)(\alpha+2)^{l-1} h_{r}(\alpha) v\right) \\
& =2\left(0+2^{l-1} c\left(v^{\top}\left(\beta_{N}+2\right)^{-1} w\right)^{l} w^{\top}(\alpha+2)^{l-1} h_{r}(\alpha) v\right) \\
& =c\left(2 v^{\top}\left(\beta_{N}+2\right)^{-1} w\right)^{l}\left(w^{\top}(\alpha+2)^{l-1} h_{r}(\alpha) v\right)
\end{aligned}
$$

Theorem 8.6

$$
\begin{aligned}
& d_{2 k+1}((2 k+1) n+1)=(2 n+1)^{k} \\
& d_{2 k}(2 k n+1)=(-1)^{k n} \\
& d_{2 k}(2 k n+k+1)=-(-1)^{k n+\left(\begin{array}{l}
k \\
2
\end{array}\right) 4^{k-1}(n+1)^{k-1}}
\end{aligned}
$$

Proof Given $w$, it is straightforward to verify the hypotheses and evaluate the final expression of Lemma 8.5 with the lemmas of Section 7 
For the first formula, take $w$ to be

$$
w_{5}=(-1)^{n}\left(\sum_{m=0}^{n}(-1)^{m} e_{(2 k+1) m}-\sum_{m=0}^{n-1}(-1)^{m} e_{(2 k+1) m+2 k}\right) \text {, }
$$

where $\left\{e_{i}\right\}_{i=0}^{N-1}$ is the standard basis.

For the second formula, $w$ to be

$$
w_{6}=(-1)^{n}\left(\sum_{m=0}^{n}(-1)^{m} e_{2 k m}-\sum_{m=0}^{n-1}(-1)^{m} e_{2 k m+2 k-1}\right) \text {. }
$$

For the third formula, use

$$
w_{7}=(-1)^{n-1}\left(\sum_{m=0}^{n}(-1)^{m} e_{2 k m+k-1}-\sum_{m=0}^{n}(-1)^{m} e_{2 k m+k}\right) .
$$

\section{Conjectures}

Let

$$
d_{r}^{\prime}(n)=\operatorname{det}\left(\frac{r}{2 i+2 j+r}\left(\begin{array}{c}
2 i+2 j+r \\
i+j
\end{array}\right)\right)_{i, j=0}^{n-1} .
$$

These sequences are considered alongside $d_{r}(n)$ in [1]. Computer experiments suggest the following conjectures:

$$
\begin{aligned}
& d_{2 k+1}^{\prime}((2 k+1) n)=d_{2 k+1}^{\prime}((2 k+1) n+1)=(-1)^{k n} \\
& d_{2 k+1}^{\prime}((2 k+1) n+k)=-d_{2 k+1}^{\prime}((2 k+1) n+k+2) \\
& =(-1)^{k n+\left(\begin{array}{c}
k \\
2
\end{array}\right)((2 k+1)(n+1))^{k-1}} \\
& d_{2 k+1}^{\prime}((2 k+1) n+k+1)=0 \\
& d_{2 k}^{\prime}(k n)=-d_{2 k}^{\prime}(k n+1)=(-1)^{n\left(\begin{array}{c}
k \\
2
\end{array}\right)(n+1)^{k-1}}
\end{aligned}
$$

Moreover, it seems that

$$
\begin{aligned}
\left(\frac{r}{2 i+2 j+r}\left(\begin{array}{c}
2 i+2 j+r \\
i+j
\end{array}\right)\right)_{i, j \geq 0} \\
= \begin{cases}A\left(-a_{k}(\gamma)(\gamma+2)^{k-1}\right) A^{\top} & \text { if } r=2 k \\
A\left((-1)^{k+1} g_{k}(-\gamma)(\gamma-2)(\gamma+2)^{k-1}\right) A^{\top} & \text { if } r=2 k+1 .\end{cases}
\end{aligned}
$$




\section{References}

1. J. Cigler. Catalan numbers, Hankel determinants and Fibonacci polynomials. January 2018. arXiv: 1801.05608.

2. J. Cigler and C. Krattenthaler. Some determinants of path generating functions. Advances in Applied Mathematics, 46(1):144 - 174, 2011.

3. Ömer Eğecioğlu, Timothy Redmond, and Charles Ryavec. A multilinear operator for almost product evaluation of Hankel determinants. Journal of Combinatorial Theory, Series A, 117(1):77 - 103, 2010. 